\title{
Calibration of the Top-Quark Monte-Carlo Mass
}

\author{
Jan Kieseler and Katerina Lipka \\ Deutsches Elektronen Synchrotron DESY, Notkestr. 85, D-22607 Hamburg, Germany \\ Sven-Olaf Moch \\ II. Institut für Theoretische Physik, Universität Hamburg, \\ Luruper Chaussee 149, D-22761 Hamburg, Germany
}

(Dated: November 4, 2015)

\begin{abstract}
We present a method to establish experimentally the relation between the top-quark mass $m_{t}^{\mathrm{MC}}$ as implemented in Monte-Carlo generators and the Lagrangian mass parameter $m_{t}$ in a theoretically well-defined renormalization scheme. We propose a simultaneous fit of $m_{t}^{\mathrm{MC}}$ and an observable sensitive to $m_{t}$, which does not rely on any prior assumptions about the relation between $m_{t}$ and $m_{t}^{\mathrm{MC}}$. The measured observable is independent of $m_{t}^{\mathrm{MC}}$ and can be used subsequently for a determination of $m_{t}$. The analysis strategy is illustrated with examples for the extraction of $m_{t}$ from inclusive and differential cross sections for hadro-production of top-quarks.
\end{abstract}

\section{INTRODUCTION}

The top-quark mass is one of the fundamental parameters of the Standard Model (SM). Its value significantly affects predictions for many observables either directly or via radiative corrections. As a consequence, the measured top-quark mass is one of the crucial inputs to electroweak precision fits, which enable comparisons between experimental results and predictions within and beyond the SM [1]. Furthermore, together with the Higgs-boson mass, it has critical implications on the stability of the electroweak vacuum 2 -4.

The top-quark mass has been determined with remarkable precision: the current world average quoted as $173.34 \pm 0.76 \mathrm{GeV}$ is obtained by combining results from the Tevatron and the LHC [5]. However, these measurements rely on the relation between the top-quark mass and the respective experimental observable, e.g., the reconstructed invariant mass of the top-quark decay products. This relation is derived from Monte Carlo (MC) simulations. Hence, these measurements determine the top-quark mass parameter implemented in this simulation, i.e., the so-called Monte-Carlo mass $m_{t}^{\mathrm{MC}}$, which appears most appropriate to describe experimental data [1, 5, 6].

In theory predictions, on the other hand, the top-quark mass appears as a parameter of the Lagrangian and, therefore, depends on the choice of the renormalization scheme once corrections beyond leading order (LO) are consistently included. The conventional scheme choice in many applications of Quantum Chromodynamics (QCD) is the pole mass $m_{t}^{\mathrm{p}}$, while alternative definitions based on the (modified) minimal subtraction realize the concept of a running mass $\bar{m}_{t}(\mu)$ at a renormalization scale $\mu$ as a particular example of so-called short-distance masses. Since MC simulations generally contain not only hardinteraction calculations at LO or next-to leading order (NLO), but also contributions from initial and final state radiation, hadronization, as well as underlying-event in- teractions, modeled by parton shower programs based on leading-logarithm approximations and heuristic models, they do not allow for a precise definition of the quark mass renormalization scheme.

The unambiguous interpretation of the experimental results for $m_{t}^{\mathrm{MC}}$ in terms of a Lagrangian top-quark mass $m_{t}$ in a specific renormalization scheme employed in the SM has been a longstanding and increasingly urgent problem, given the importance of the value of the top-quark mass for SM physics analysis and the small uncertainty in the experimental measurement of $m_{t}^{\mathrm{MC}}[5]$. At present, the translation from $m_{t}^{\mathrm{MC}}$ to a theoretically well-defined mass definition in a short-distance scheme at a low scale can only be estimated to be $\mathcal{O}(1) \mathrm{GeV}$, see, e.g., Ref. [7, 8].

In consequence, a measurement of $m_{t}$ is preferable and can be performed by confronting a measured observable sensitive to $m_{t}$ with its prediction, calculated at $\mathrm{NLO}$ in QCD or beyond in a well-defined renormalization scheme for the top-quark mass. For this purpose, inclu-

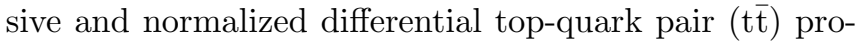
duction cross sections have been employed to determine the pole mass 9 11. For these measurements of $m_{t}^{\mathrm{p}}$, detector and process modeling effects are evaluated using MC simulations, so that the measured observable typically depends on $m_{t}^{\mathrm{MC}}$. Even though the extracted value of $m_{t}^{\mathrm{p}}$ does not depend on a specific $m_{t}^{\mathrm{MC}}$ hypothesis, it relies on the relation between both parameters, the exact difference $\left(\Delta_{m}=m_{t}^{\mathrm{p}}-m_{t}^{\mathrm{MC}}\right)$ being unknown 911 and leading to a systematic uncertainty on the measurement. This uncertainty can be negligible when only the shape of a particular observable defined within the detectors fiducial volume is considered [11, since the dependence on $m_{t}^{\mathrm{MC}}$ mainly enters through detector-acceptance effects. However, the sensitivity to $m_{t}$ increases when the total $\mathrm{t} \overline{\mathrm{t}}$ production rate is also taken into account.

This letter describes a generic approach to measure an observable $\xi$ sensitive to $m_{t}$ without any prior assumptions on $m_{t}^{\mathrm{MC}}$ or its relation to $m_{t}$. The method employs 
a simultaneous likelihood fit of $m_{t}^{\mathrm{MC}}$ and $\xi$, comparing an observed distribution in data to its MC prediction. For the latter, two categories of processes are taken into account: the signal process, for which production cross section and event kinematics depend on $m_{t}$, and background processes with no or negligible dependence on $m_{t}$. Subsequently, one can perform a determination of $m_{t}$ in a given renormalization scheme comparing to theory predictions for $\xi\left(m_{t}\right)$ and, therefore, a calibration of $m_{t}^{\mathrm{MC}}$ by quantifying the difference $\Delta_{m}=m_{t}-m_{t}^{\mathrm{MC}}$. The method is first discussed for the special case with $\xi$ being an inclusive signal production cross section and extended to differential cross sections in a second step.

\section{INCLUSIVE CROSS SECTIONS}

To measure the inclusive cross section $\sigma, N^{d}$ events are reconstructed and selected experimentally, with an efficiency $\epsilon$ estimated from simulation. In total $N^{p}$ expected events are confronted with those observed in data in bins of an observable sensitive to $m_{t}^{\mathrm{MC}}$. The parameterization is chosen such that the shape of the distribution constrains $m_{t}^{\mathrm{MC}}$, while its normalization determines $\sigma$. For this purpose, the fraction of predicted signal events $n_{i}^{p}$ in bin $i$ is considered and the total number of predicted events $N_{i}^{p}$ in the same bin is written as:

$$
N_{i}^{p}=\mathcal{L} \cdot \epsilon\left(m_{t}^{\mathrm{MC}}, \vec{\lambda}\right) \sigma \cdot n_{i}^{p}\left(m_{t}^{\mathrm{MC}}, \vec{\lambda}\right)+N_{i}^{b g}(\vec{\lambda}),
$$

with $N_{i}^{b g}$ being the contribution from background processes and $\mathcal{L}$ the integrated luminosity. Potential systematic uncertainties due to detector effects as well as signal and background process modeling are symbolized as parameters $\vec{\lambda}$ and affect the expected event yields. For each bin $i$, a Poisson likelihood $P$ is derived from $N_{i}^{p}$ and the number of observed events $N_{i}^{d}$. The values for $\sigma$ and $m_{t}^{\mathrm{MC}}$ are determined from the maximum $L_{\max }$ of the global likelihood

$$
L\left(\sigma, m_{t}^{\mathrm{MC}}, \vec{\lambda}\right)=\prod_{i} P\left(N_{i}^{p}\left(\sigma, m_{t}^{\mathrm{MC}}, \vec{\lambda}\right), N_{i}^{d}\right) \cdot \Xi(\vec{\lambda}) .
$$

Here, $\Xi(\vec{\lambda})$ represents optional terms that can model prior knowledge on the systematic uncertainties specific to the experiment. Alternatively, the fit can be repeated for each individual systematic variation, leaving only $m_{t}^{\mathrm{MC}}$ and $\sigma$ as free parameters.

Explicit correlations between $\sigma$ and $m_{t}^{\mathrm{MC}}$ are introduced by the term $\epsilon\left(m_{t}^{\mathrm{MC}}, \vec{\lambda}\right)$. Hence, the contribution of $m_{t}^{\mathrm{MC}}$ to the total uncertainty on $\sigma$ can be minimized by reducing the dependence of $\epsilon$ on $m_{t}^{\mathrm{MC}}$ or by the strong constraints on $m_{t}^{\mathrm{MC}}$ through $n_{i}^{p}$. For an optimal calibration of $m_{t}^{\mathrm{MC}}$ to $m_{t}$, both effects should be combined.

The predicted cross section $\sigma^{p}$ is expressed as a function of $m_{t}$. With $\sigma$ being independent of $m_{t}^{\mathrm{MC}}, m_{t}$ can be determined directly from the value for which the predicted and measured cross sections coincide. Uncertainties on $m_{t}$ are evaluated using error propagation. For the uncertainty on the calibration of $m_{t}^{\mathrm{MC}}$ to $m_{t}$, the correlations between $\sigma$ and $m_{t}^{\mathrm{MC}}$ need to be accounted for and are known precisely as a result of the simultaneous fit. The contribution from the statistical uncertainty on $\sigma$ and $m_{t}^{\mathrm{MC}}$ might be underestimated, since fluctuations due to limited statistics in the MC simulation of the signal are not taken into account in the likelihood. However, the effect can be evaluated using pseudo experiments.

Precise measurements of the inclusive t $\bar{t}$ cross section are performed in the dileptonic decay channel by the ATLAS and CMS collaborations [9, 10]. The uncertainties of these measurements are below $4 \%$ and the dependence on $m_{t}^{\mathrm{MC}}$ is small. The precision is mainly limited by variations of global normalization parameters such as the luminosity, while jet energy scale and jet modeling uncertainties are constrained. In both analyses, $m_{t}^{\mathrm{p}}$ is extracted assuming $\left|\Delta_{m}\right| \lesssim 1 \mathrm{GeV}$, and assigning a corresponding uncertainty. The resulting total precision of $m_{t}^{\mathrm{p}}$ is about $2 \mathrm{GeV}\left[9\right.$. Measurements of $m_{t}^{\mathrm{MC}}$ have been performed in the same t $\bar{t}$ decay channel using LHC data at a center-of-mass energy of $\sqrt{s}=7$ or $8 \mathrm{TeV}[12,13$. The value of $m_{t}^{\mathrm{MC}}$ is extracted from the normalized distribution of the lepton and b-jet invariant mass $m_{l b}$. The resulting precision is about $1.3 \mathrm{GeV}$ and in this case mostly affected by uncertainties on the jet energy scale and modeling. When combining these analyses of $m_{t}^{\mathrm{MC}}$ and $\sigma$ as proposed in this letter, the correlation between the measured $\sigma$ and $m_{t}^{\mathrm{MC}}$ would therefore become small. In addition, a reduction of the uncertainty on $m_{t}^{\mathrm{MC}}$ could be achieved without increasing the uncertainty on $\sigma$. The details of the implementation depend on the particular analysis. Also additional observables can be considered to constrain systematic uncertainties in a simultaneous fit as in Ref. 9], but statistical correlations between the bins must be avoided by considering each event only once.

The obtained value of $\sigma$ can be used to extract the pole mass $m_{t}^{\mathrm{p}}$ independently of its relation to $m_{t}^{\mathrm{MC}}$ and to determine $\Delta_{m}$. However, the pole mass scheme, which is inspired by the definition of the electron mass in Quantum Electrodynamics, has short-comings when applied to quarks in a confined theory [14, 15]. Non-perturbative corrections to $m_{t}^{\mathrm{p}}$ due to the infrared renormalon lead to an intrinsic theoretical ambiguities of the order of $\Lambda_{\mathrm{QCD}}$ [14 16. Alternatively, $\sigma$ can be calculated using other mass schemes [17 20], such as the aforementioned running mass definition at a scale $\mu, \bar{m}_{t}(\mu)$, the so-called $\overline{\mathrm{MS}}$ mass. Since $\sigma$ is measured independently of $\Delta_{m}$, also $\bar{m}_{t}$ can be determined directly. By using $\bar{m}_{t}$ in the calculation of $\sigma$, the perturbative expansion in the strong coupling exhibits a significantly faster convergence [20. This fact has two implications: the extracted $\bar{m}_{t}$ will be more precise than $m_{t}^{\mathrm{p}}$ obtained at the same order of per- 
turbation theory and additional higher-order corrections will result in smaller corrections to $\bar{m}_{t}$ than $m_{t}^{\mathrm{p}}$.

A detailed experimental analysis employing the method proposed here is documented in Ref. [21. For illustration, the $t \overline{\mathrm{t}}$ production cross section measured in Ref. [10] at $\sqrt{s}=8 \mathrm{TeV}$ for $m_{t}^{\mathrm{MC}}=172.5 \mathrm{GeV}$ as $\sigma=242.4 \pm 9.5 \mathrm{pb}$ is used to determine $\bar{m}_{t}$ and $m_{t}^{\mathrm{p}}$. The value and uncertainty on $\sigma$ is assumed to be independent of $m_{t}$. The LHC beam-energy uncertainty of $1.72 \%$ is assigned to the predicted cross section, evaluated with the program HATHOR 22 relying on calculations of Refs. [20, 23, 26]. The cross section is calculated at LO, NLO, and next-to-next-to leading order (NNLO) accuracy with the strong coupling constant $\alpha_{S}$ at the Zboson mass $M_{Z}$ set to $\alpha_{S}\left(M_{Z}\right)=0.118 \pm 0.001$ and based on the parton distribution (PDF) set CT14 [27] evaluated at NNLO. Renormalization and factorization scales are set to $m_{t}^{\mathrm{p}}$ or $\bar{m}_{t}$, respectively, and varied independently by a factor of 2 up and down. The uncertainties due to variations of the CT14 PDF eigenvectors are scaled to $68 \%$ confidence level.

The extraction of $m_{t}^{\mathrm{p}}$ and $\bar{m}_{t}$ is performed by comparison of predicted and measured $\sigma$. Experimental and theoretical uncertainties are considered uncorrelated. The resulting top-quark mass values are illustrated in Fig. 1. Predictions at LO cannot fix the mass scheme. When higher orders are accounted for in the calculation of $\sigma$, $\bar{m}_{t}$ exhibits a more rapid convergence than $m_{t}^{\mathrm{p}}$. Moreover, the variations of the renormalization and factorization scales affect the prediction of $\sigma$ expressed in terms of $\bar{m}_{t}$ less than for $m_{t}^{\mathrm{p}}$, resulting in a smaller total uncertainty on $\bar{m}_{t}$ than on $m_{t}^{\mathrm{p}}$ with $\pm_{2.2}^{2.1} \mathrm{GeV}$ and $\pm_{2.9}^{2.4} \mathrm{GeV}$ at NNLO, respectively.

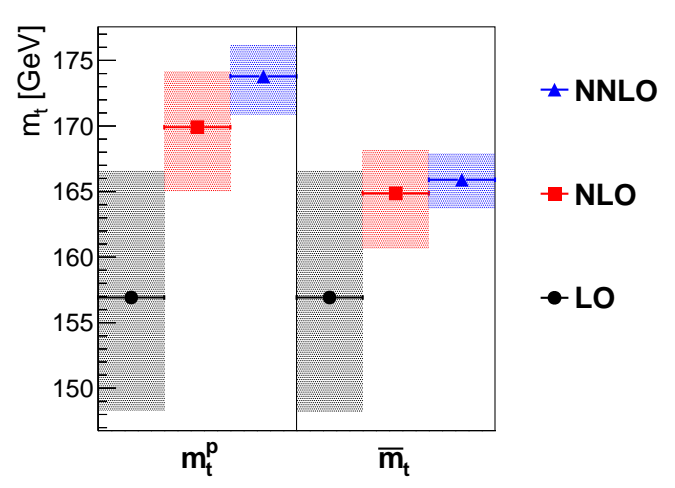

FIG. 1. Top-quark pole $\left(m_{t}^{\mathrm{p}}\right)$ and $\overline{\mathrm{MS}}$ mass $\left(\bar{m}_{t}\right)$ extracted from the inclusive t $\bar{t}$ production cross section by comparison with its prediction at different orders of perturbative QCD. The hatched areas indicate the total uncertainty on the measured mass values.

The obtained $\overline{\mathrm{MS}}$ mass $\bar{m}_{t}$ can be converted to the pole mass $m_{t}^{\mathrm{p}}$ in perturbation theory with up to four-loop accuracy in QCD 28. It is well-known that this leads to additional positive shifts of the value of $m_{t}^{\mathrm{p}}$, the size of which indicates the residual theoretical uncertainty $m_{t}^{\mathrm{p}}$ at higher orders. E.g., using a fixed $\bar{m}_{t}$ as input, the value of $m_{t}^{\mathrm{p}}$ is approximately $0.5 \mathrm{GeV}(0.2 \mathrm{GeV})$ larger if the conversion formula is applied at three(four)-loop instead of two(three)-loop accuracy, respectively.

All extracted top-quark mass values in well-defined schemes can be used to calibrate the $m_{t}^{\mathrm{MC}}$ parameter, which is non universal and, in principle, depends on the subtleties of its implementation in the MC simulation. In a given renormalization scheme the difference $m_{t}-m_{t}^{\mathrm{MC}}$ can be quantified fully consistently, and, with the current precision of the measured and predicted $t \bar{t}$ production cross sections and $m_{t}^{\mathrm{MC}}$ measurements, an uncertainty of approximately $2 \mathrm{GeV}$ on this calibration can be expected, depending on the correlations between the parameters.

\section{DIFFERENTIAL CROSS SECTIONS}

An extension of the method to differential cross sections used for the determination of $m_{t}$ can provide a larger sensitivity and, possibly, a further reduction of systematic uncertainties. In the following, a differential production cross section for the signal process as a function of an observable $x$ is considered and employed to determine $m_{t}$. The approach used for $\sigma$ is applied to each bin of this differential cross section. For this purpose, the efficiency $\epsilon$ is replaced by a matrix $M$ describing the detector response to the predicted cross section $\sigma_{k}^{\mathrm{MC}}$ in bin $k$ of the distribution in terms of $x$, defined by:

$$
N_{j}^{p s}=\mathcal{L} \cdot \sum_{k} M_{j k} \sigma_{k}^{\mathrm{MC}}
$$

with $N_{j}^{p s}$ being the predicted number of reconstructed and selected signal events in bin $j$ of the reconstructed distribution. The response matrix is derived from MC simulation and therefore depends on $\vec{\lambda}$ as well as on $m_{t}^{\mathrm{MC}}[29$.

Each bin $j$ of the reconstructed distribution is considered as a category. In each category, a second observable $y$ is defined, sensitive to $m_{t}^{\mathrm{MC}}$. The shape of this observable is used to constrain $m_{t}^{\mathrm{MC}}$, while the total number of signal events in each category corresponds to $N_{j}^{p s}$, and hence can be used to derive the differential cross section. The number of predicted events, $N_{i j}^{p}$, in bin $i$ of the observable $y$ is given as:

$N_{i j}^{p}=\mathcal{L} \cdot \sum_{k} M_{j k}\left(m_{t}^{\mathrm{MC}}, \vec{\lambda}\right) \sigma_{k}^{\mathrm{MC}} \cdot n_{i j}^{p}\left(m_{t}^{\mathrm{MC}}, \vec{\lambda}\right)+N_{i j}^{b g}(\vec{\lambda})$,

with $n_{i j}^{p}$ being the fraction of predicted signal events in bin $i$ with respect to $N_{j}^{p s}$ and $N_{i j}^{b g}$ the contribution from background processes.

By comparison with the number of observed events $N_{i j}^{d}$ in each category $j$ and bin $i$, and considering $\sigma_{k}^{\mathrm{MC}} \rightarrow \sigma_{k}$ 
as free parameters a fit can be performed maximizing the likelihood:

$$
L\left(\sigma_{0}, \ldots, \sigma_{k}, m_{t}^{\mathrm{MC}}, \vec{\lambda}\right)=\prod_{i} \prod_{j} P\left(N_{i j}^{p}, N_{i j}^{d}\right) \cdot \Xi(\vec{\lambda}) .
$$

This unfolding problem can be ill-posed and regularization techniques might need to be applied. A well-suited regularization condition is provided, for instance, by the aim to determine $m_{t}$ by comparison of $\sigma_{k}$ with its prediction $\sigma_{k}^{p}\left(m_{t}\right)$ as a function of $m_{t}$. Replacing $\sigma_{k}$ with this prediction corresponds to the folding approach used in Ref. [12 and reduces the number of free parameters significantly, such that the likelihood becomes:

$$
L\left(m_{t}, m_{t}^{\mathrm{MC}}, \vec{\lambda}, \vec{\kappa}\right)=\prod_{i} \prod_{j} P\left(N_{i j}^{p}, N_{i j}^{d}\right) \cdot \Xi(\vec{\lambda}, \vec{\kappa}),
$$

with $\Xi(\vec{\lambda}, \vec{\kappa})$ representing optional nuisance terms and $\vec{\kappa}$ being theoretical uncertainties on the predicted $\sigma_{k}^{p}\left(m_{t}\right)$. Both, $\vec{\lambda}$ and $\vec{\kappa}$ can be incorporated as nuisance terms in $\Xi$ or can be evaluated individually. In the latter case, $L$ depends on $m_{t}$ and $m_{t}^{\mathrm{MC}}$, only. A maximization of $L$ directly returns the relation between these parameters as well as their correlations. The correlations are mainly incorporated through the response matrix $M$. Therefore, the event selection and the observable $x$ should be chosen such, that the dependence of $M$ on $m_{t}^{\mathrm{MC}}$ is minimized and the sensitivity of $y$ on $m_{t}^{\mathrm{MC}}$ becomes maximal.

For the optimization of the result, also the correlation between the observables $x$ and $y$ should be small. A possible choice for $x$ would be the differential t $\overline{\mathrm{t}}$ production cross section as a function of the top-quark transverse momentum predicted up to NNLO accuracy [30]. The dependence of this observable on $m_{t}^{\mathrm{p}}$ and $\bar{m}_{t}$ can be studied at approximate NNLO with programs publicly available 31. This distribution, describing the production dynamics, can be combined with an observable based on the kinematics of the decay products such as $m_{l b}$ in the dileptonic decay channel or the invariant mass of the 3 jets that originate from the top-quark decay $t \rightarrow W b \rightarrow b q \bar{q}$ in the semileptonic channel.

The additional sensitivity of the differential cross sections to $m_{t}$ can result in uncertainties below $2 \mathrm{GeV}$ on $m_{t}$ and $\Delta_{m}$, starting to challenge the measurements of $m_{t}^{\mathrm{MC}}$ in precision and improving the understanding of this parameter. Moreover, a simultaneous determination of the strong coupling $\alpha_{S}$ and $m_{t}$ becomes possible.

\section{CONCLUSION}

The simultaneous determination of $m_{t}^{\mathrm{MC}}$ and differential or inclusive production cross sections of processes sensitive to the top-quark mass $m_{t}$ with subsequent extraction of $m_{t}$ in a well-defined renormalization scheme from those measured cross sections solves the longstanding problem of the calibration of the top-quark Monte Carlo mass $m_{t}^{\mathrm{MC}}$. The proposed method, therefore, allows to consistently quantify the difference $\Delta_{m}=m_{t}-$ $m_{t}^{\mathrm{MC}}$ within the uncertainties of the measurement.

The extraction of $m_{t}$ is preferably performed in a scheme, where the perturbative expansion of the theory prediction for the respective cross section displays fast apparent convergence. This applies to short-distance masses and favors an experimental determination of a running top-quark mass $\bar{m}_{t}$ over the pole mass $m_{t}^{\mathrm{p}}$. The latter can always be obtained from conversion formula up to four-loop accuracy in QCD.

With the current precision of the inclusive top-quark cross-section and mass measurements an uncertainty on $\Delta_{m}$ of approximately $2 \mathrm{GeV}$ is expected. Dedicated analyses based on differential cross sections seem to be a promising approach to further decrease these uncertainties and to measure theoretically well-defined mass parameters independently of the top-quark MC mass to a high precision.

\section{Acknowledgments}

We would like to thank Olaf Behnke for useful discussions.

[1] K. A. Olive et al. (Particle Data Group), Chin. Phys. C38, 090001 (2014)

[2] F. Bezrukov, M. Yu. Kalmykov, B. A. Kniehl, and M. Shaposhnikov, JHEP 10, 140 (2012), arXiv:1205.2893 [hep-ph]

[3] G. Degrassi, S. Di Vita, J. Elias-Miro, J. R. Espinosa, G. F. Giudice, et al., JHEP 1208, 098 (2012), arXiv:1205.6497 [hep-ph].

[4] S. Alekhin, A. Djouadi, and S. Moch, Phys. Lett. B716, 214 (2012), arXiv:1207.0980 [hep-ph].

[5] (ATLAS and CDF and CMS and D0 Collaborations), (2014), arXiv:1403.4427 [hep-ex]

[6] A. Buckley, J. Butterworth, S. Gieseke, D. Grellscheid, S. Höche, et al., Phys. Rept. 504, 145 (2011) arXiv:1101.2599 [hep-ph].

[7] A. H. Hoang and I. W. Stewart, Nucl. Phys. Proc. Suppl. 185, 220 (2008), arXiv:0808.0222 [hep-ph]

[8] S. Moch et al., (2014), arXiv:1405.4781 [hep-ph].

[9] (CMS Collaboration), Measurement of the tt production cross section in the e $\mu$ channel in $p p$ collisions at 7 and $8 \mathrm{TeV}$, Tech. Rep. CMS-PAS-TOP-13-004 (CERN, Geneva, 2015).

[10] G. Aad et al. (ATLAS Collaboration), Eur. Phys. J. C74, 3109 (2014), arXiv:1406.5375 [hep-ex]

[11] G. Aad et al. (ATLAS Collaboration), JHEP 10, 121 (2015), arXiv:1507.01769 [hep-ex]

[12] (CMS Collaboration), Determination of the top-quark mass from the $m(l b)$ distribution in dileptonic tt events at 
$\sqrt{s}=8 \mathrm{TeV}$, Tech. Rep. CMS-PAS-TOP-14-014 (CERN, Geneva, 2014).

[13] G. Aad et al. (ATLAS Collaboration), Eur. Phys. J. C75, 330 (2015), arXiv:1503.05427 [hep-ex]

[14] I. I. Y. Bigi, M. A. Shifman, N. G. Uraltsev, and A. I. Vainshtein, Phys. Rev. D50, 2234 (1994), arXiv:hepph/9402360 [hep-ph].

[15] M. Beneke and V. M. Braun, Nucl. Phys. B426, 301 (1994), arXiv:hep-ph/9402364 [hep-ph],

[16] M. C. Smith and S. S. Willenbrock, Phys. Rev. Lett. 79, 3825 (1997), arXiv:hep-ph/9612329 [hep-ph]

[17] A. H. Hoang, Z. Ligeti, and A. V. Manohar, Phys. Rev. D59, 074017 (1999), arXiv:hep-ph/9811239 [hep-ph]

[18] A. H. Hoang and T. Teubner, Phys. Rev. D60, 114027 (1999), arXiv:hep-ph/9904468 [hep-ph],

[19] M. Beneke, Phys. Lett. B434, 115 (1998), arXiv:hep$\mathrm{ph} / 9804241$ [hep-ph].

[20] U. Langenfeld, S. Moch, and P. Uwer, Phys. Rev. D80, 054009 (2009), arXiv:0906.5273 [hep-ph]

[21] J. Kieseler, Measurement of Top-Quark Pair Production Cross Sections and Calibration of the Top-Quark MonteCarlo Mass using LHC Run I Proton-Proton Collistion Data at $\sqrt{s}=7$ and $8 \mathrm{TeV}$ with the CMS Experiment,
Tech. Rep. to appear (DESY, Hamburg, 2015).

[22] M. Aliev, H. Lacker, U. Langenfeld, S. Moch, P. Uwer, et al., Comput. Phys. Commun. 182, 1034 (2011), arXiv:1007.1327 [hep-ph].

[23] P. Bärnreuther, M. Czakon, and A. Mitov, Phys. Rev. Lett. 109, 132001 (2012), arXiv:1204.5201 [hep-ph]

[24] M. Czakon and A. Mitov, JHEP 1212, 054 (2012), arXiv:1207.0236 [hep-ph].

[25] M. Czakon and A. Mitov, JHEP 1301, 080 (2013), arXiv:1210.6832 [hep-ph]

[26] M. Czakon, P. Fiedler, and A. Mitov, Phys. Rev. Lett. 110, 252004 (2013), arXiv:1303.6254 [hep-ph],

[27] S. Dulat, T. J. Hou, J. Gao, M. Guzzi, J. Huston, et al., (2015), arXiv:1506.07443 [hep-ph].

[28] P. Marquard, A. V. Smirnov, V. A. Smirnov, and M. Steinhauser, Phys. Rev. Lett. 114, 142002 (2015), arXiv:1502.01030 [hep-ph].

[29] A more complete discussion of the response matrix can be found in Ref. 12 .

[30] M. Czakon, D. Heymes, and A. Mitov, (2015), arXiv:1511.00549 [hep-ph].

[31] M. Guzzi, K. Lipka, and S. Moch, JHEP 01, 082 (2015), arXiv:1406.0386 [hep-ph] 